\title{
DEGAS 2 Neutral Transport Modeling of High Density, Low Temperature Plasmas
}

\author{
D. P. Stotler, A. Yu. Pigarov, ${ }^{* \dagger}$ C. F. F. Karney, S. I. Krasheninnikov, ${ }^{* \dagger}$ \\ B. LaBombard, ${ }^{*}$ B. Lipschultz, ${ }^{*}$ G. M. McCracken, ${ }^{*}$ A. Niemczewski, ${ }^{* \ddagger}$ \\ J. A. Snipes, ${ }^{*}$ J. L. Terry, ${ }^{*}$ and R. A. Vesey ${ }^{\S}$ \\ Plasma Physics Laboratory, Princeton University \\ P.O. Box 451, Princeton, NJ 08543 USA
}

\begin{abstract}
Neutral transport in the high density, low temperature plasma regime is examined using the DEgAs 2 Monte Carlo neutral transport code. Degas 2 is shown to agree with an analytic fluid neutral model valid in this regime as long as the grid cell spacing is less than twice the neutral mean-free path. Using new atomic physics data provided by the collisional radiative code CRAMD, DEGAS 2 is applied to a detached Alcator C-Mod discharge. A model plasma with electron temperature $\sim 1 \mathrm{eV}$ along detached flux tubes, between the target and the ionization front, is used to demonstrate that recombination is essential to matching the experimental data. With the CRAMD data, $\sim 20 \%$ of the total recombination is due to molecular activated recombination.
\end{abstract}

\footnotetext{
*Massachusetts Institute of Technology, Plasma Fusion Center, 167 Albany Street, Cambridge, MA 02139, USA

${ }^{\dagger}$ Also at I. V. Kurchatov Institute of Atomic Energy 1 Kurchatov Sq., Moscow 123098, Russia

${ }^{\ddagger}$ Presently at McKinsey \& Company, Inc., London SW1Y 4UH, United Kingdom

${ }^{\S}$ Presently at Sandia National Laboratories P.O. Box 5800, Albuquerque, NM, USA
} 


\section{DISCLAIMER}

This report was prepared as an account of work sponsored by an agency of the United States Government. Neither the United States Government nor any agency thereof, nor any of their employees, make any warranty, express or implied, or assumes any legal liability or responsibility for the accuracy, completeness, or usefulness of any information, apparatus, product, or process disclosed, or represents that its use would not infringe privately owned rights. Reference herein to any specific commercial product, process, or service by trade name, trademark, manufacturer, or otherwise does not necessarily constitute or imply its endorsement, recommendation, or favoring by the United States Government or any agency thereof. The views and opinions of authors expressed herein do not necessarily state or reflect those of the United States Government or any agency thereof. 


\section{DISCLAIMER}

Portions of this document may be illegible in electronic image products. Images are produced from the best available original document. 


\section{INTRODUCTION}

Detaching the plasma from the divertor target has been proposed as a method of power dispersal in the ITER[1] divertor. Typical plasma conditions in the detached state are electron temperatures $\sim 1 \mathrm{eV}$, electron densities $\sim 10^{21}$ $\mathrm{m}^{-3}$, and neutral densities $\sim 10^{20} \mathrm{~m}^{-3}$. Under these conditions, the chemical reactions involving hydrogen atoms, molecules, and ions are tightly coupled.

The short mean-free path. neutral transport and atomic physics reactions used in DEGAS 2[2] must be accurately represented if the detached plasma sources, sinks and radiation are to be computed reliably. To this end, we benchmark the neutral transport in DEGAS 2 against an analytic model of a partially ionized fluid plasma[3]. In addition, the collisional-radiative atomic physics code CRAMD[4] has been developed in parallel with DEGAS 2 for the purpose of computing the effective ionization, recombination, dissociation, and radiation rates for these high density, low temperature plasmas. Initial applications to a partially detached Alcator C-Mod[5] discharge will be described.

\section{COMPARISON WITH FLUID MODEL}

The fluid equations in Ref. [3], derived under the assumption of a constant charge exchange cross section, contain terms representing the thermal force and diffusion thermoeffect. Neglecting viscosity, ionization, and recombination, the resulting fluid neutral momentum balance in a slab geometry is

$$
\frac{d}{d x}\left(m n v^{2}+n T\right)=\alpha_{T} n \frac{d T}{d x}-m \nu_{\mathrm{cx}} n v
$$

where $n$ is the neutral density, $v$ its flow velocity, $T$ is its temperature (assumed equal to the ion temperature $T_{i}$ ), and $m$ is the ion and neutral mass. The thermal force coefficient, $\alpha_{T}=0.24$, and the charge exchange frequency, $\nu_{\mathrm{cx}}=$ $2.93 \sigma_{\mathrm{cx}} n(T / m)^{1 / 2}$.

With a neutral source on one end of the slab $(x=0)$ and an exit at the other $(x=L)$, an approximate analytic solution to Eq. (1) can be obtained by writing the exiting flux as $j=\gamma n(L)[T(L) / m]^{1 / 2}$, where $\gamma \sim 1$ is an undetermined numerical factor. The results are insensitive to the precise value of $\gamma$. Equation (1) can be written in terms of the relative density $\eta \equiv n(x) / n(L)$,

$$
\frac{d \eta}{d x}=-\left[\frac{\frac{\left(1-\alpha_{T}\right)}{T(L)} \frac{d T(x)}{d x} \eta+\frac{\gamma \nu_{\mathrm{cx}}}{[T(L) / m]^{1 / 2}}}{\frac{T(x)}{T(L)}-\frac{\gamma^{2}}{\eta^{2}}}\right],
$$

and numerically integrated from $x=L$ to $x=0$ using specified plasma temperature profiles.

The critical parameters in the comparison between Eq. (1) and DEGAS 2 are the mean-free path to system size ratio $l / L=[T(L) / m]^{1 / 2} /\left(L \nu_{\mathrm{cx}}\right)$ and the mean-free path to grid spacing ratio $l / \Delta$, where $\Delta$ is the width of the individual DEGAS 2 grid cells. If $l / L$ is too big, the neutrals and ions are not well coupled, 


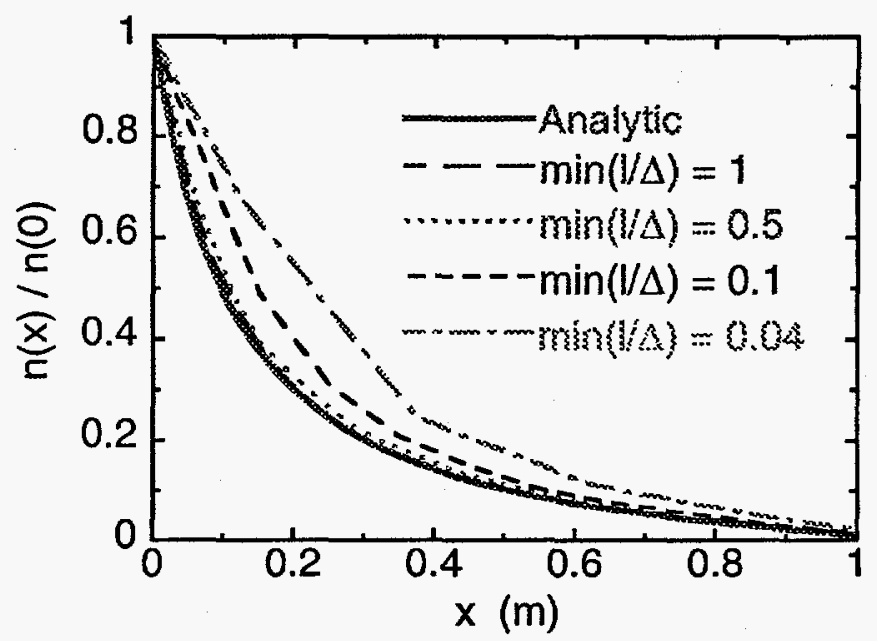

Figure 1: Comparison between four DEGAS 2 simulations at different mean-free path to grid spacing ratios and an analytic solution [Eq. (2)] of the normalized neutral density variation with distance in a slab geometry.

and Eq. (1) is not valid. If $l / \Delta$ is too small, the DEGAS 2 neutrals do not experience enough temperature variation to resolve the thermal force.

Simulations exploring the parameter space indicate agreement between DEGAS 2 and Eq. (2) when $\max (l / L) \leq 0.1$ (i.e., maximum value of $l / L$ in the plasma) and $\min (l / \Delta) \geq 0.5$. Figure 1 demonstrates the latter inequality with four runs, all with $\max (l / L)=0.1$, and $\min (l / \Delta)=0.04,0.1,0.5$, and 1 .

\section{ATOMIC PHYSICS MODELS}

Ions striking the divertor targets in DEGAS 2 are assumed to reenter the plasma either as reflected atoms or desorbed molecules[6]. The fraction reflected and their energies are taken from experimental data and surface physics simulations[6].

For temperatures in excess of a few $\mathrm{eV}$, molecules predominantly undergo dissociation and ionization. The original version of DEGAS[6] explicitly incorporated these reactions using rates taken from the literature. Atomic $\mathrm{H}$ ionization and $\mathrm{H}^{+}$recombination were treated with a collisional-radiative calculation. This atomic physics model ("model I") is available in DEGAS 2 as well.

At temperatures below a few $\mathrm{eV}$, the rates of dissociation and ionization decrease, and vibrational excitation, dissociative attachment $\left[\mathrm{H}_{2}(v)+\mathrm{e} \rightarrow \mathrm{H}^{-}\right.$ $+\mathrm{H}]$, and ion conversion $\left[\mathrm{H}_{2}(v)+\mathrm{H}^{+} \rightarrow \mathrm{H}_{2}^{+}+\mathrm{H}\right]$ become dominant [4]. Product ions of the last two reactions rapidly recombine into two neutral atoms via $\mathrm{H}^{-}+$ $\mathrm{H}^{+} \rightarrow 2 \mathrm{H}$ and $\mathrm{H}_{2}^{+}+\mathrm{e} \rightarrow 2 \mathrm{H}$. Through this "molecular activated recombination" (MAR), the break-up of a single molecule effectively results in a source of three 
atoms and a sink of one ion.

The CRAMD package[4] aims to incorporate all relevant processes involving the ground and excited states of atoms, molecules, and their ions into a self-consistent, multispecies, collisional-radiative calculation. The resulting effective rates for hydrogen molecule break-up, atom ionization, and radiative and three-body recombination can be used directly by DEGAS 2 to compute the neutral transport of hydrogen atoms and molecules ("model II").

Model II incorporates newer cross sections for transitions between the various hydrogen atomic excited states, as well as a more careful treatment of near continuum states. For $T_{e}=5 \mathrm{eV}$, the effective ionization rates in model II are a factor of 1.6 smaller than those in model $\mathrm{I}$. At $1 \mathrm{eV}$, the recombination rate in model $\mathrm{II}$ is a factor of 1.4 smaller.

\section{DIVERTOR PLASMA MODELS}

As in Ref. [7], radial plasma density and temperature profiles in Alcator C-Mod are taken from a fast scanning probe in the main scrape-off layer and domed probes in the divertor targets. The ion fluxes to the targets are obtained from the measured parallel ion fluxes using the magnetic field line angle of incidence[7] as computed from the equilibrium. A pure deuterium plasma is assumed with $n_{i}=n_{e} \equiv n$ and $T_{i}=T_{e} \equiv T$.

In the baseline plasma model, "model $A$ ", an analytic procedure[8] is used to interpolate the plasma parameters onto the computational mesh cells[7] between the probe locations. In this model[8], the temperature profile along each flux tube is the solution of the parallel heat conduction equation with a delta-function radiation sink at $T=T_{\mathrm{rad}}=8 \mathrm{eV}$ and uniform heat source from the core plasma. Parallel ion momentum loss due to charge-exchange is assumed to occur at a constant rate for $T<T_{\mathrm{cx}}=5 \mathrm{eV}$.

Plasma "model B" is a limiting case in which we set the $T$ gradient to zero[9] along detached flux tubes between the target and the point where $T=T_{\text {rad }}$; otherwise it the same as model A. Detached flux tubes are identified as having a factor of four or more drop in the measured isotropic pressure $P$ from upstream to the plate. In this model, a factor of four decrease in pressure is assumed to occur at $T=T_{\text {rad }}$. Any further reduction is spread linearly between there and the plate. The density is determined from $n=P /(2 T)$.

Recent work[10] has suggested that the bulk electron temperatures in divertor plasmas may be lower than are indicated by the probe data. We consider a variant of model $B$, plasma "model $C$ ", in which the plate temperatures are multiplied by 0.5 before the profiles are defined. The plate densities are modified to maintain the same particle fluxes as the other two cases.

\section{DEGAS 2 SIMULATIONS}

The results of the six runs obtained with the two physics models and three plasma models of Alcator C-Mod shot 950308012 are summarized in Table I. 
At the simulated timeslice, $t=0.96 \mathrm{~s}$, the plasma is partially detached along the outer target with measured temperatures there of $1-2 \mathrm{eV}$.

The experimentally observed volume-integrated Balmer- $\alpha$ emission rate $\mathrm{D}_{\alpha}$ and divertor neutral gas pressure[9] $P_{g}$ are included in Table $I$ for comparison with the DEGAS 2 results. Since the "gas box" containing the neutral pressure gauge is not simulated, we use the $\mathrm{D}$ and $\mathrm{D}_{2}$ densities $\left(n_{\mathrm{D} \text {,div }}, n_{\mathrm{D}_{2} \text {,div }}\right)$ and pressures at the bottom of the divertor, where the entrance to the gas box would be, to compute the total neutral flux. $P_{g}$ is found by then assuming that the same flux of room temperature molecular gas reaches the gauge[9]. The effective atom temperature at the bottom of the divertor $T_{\mathrm{D} \text {,div }}$ varies with the background plasma and not with atomic physics model. It is, thus, governed more by charge exchange and wall reflection processes than molecular dissociation.

Table I: RESULTS FROM DEGAS 2 RUNS WITH THREE PLASMA MODELS (A, B, C) AND TWO ATOMIC PHYSICS MODELS (I, II)

\begin{tabular}{|c|c|c|c|c|c|c||c|}
\hline & A.I & A.II & B.I & B.II & C.I & C.II & Meas. \\
\hline \hline $\mathrm{D}_{\alpha}\left(10^{21} \mathrm{~s}^{-1}\right)$ & 1.73 & 1.17 & 2.63 & 2.01 & 33.6 & 26.4 & 8.87 \\
\hline$P_{g}\left(\mathrm{mTorr}^{\mathrm{T}}\right)$ & 0.57 & 0.57 & 1.4 & 1.6 & 26. & 23. & 32.2 \\
\hline$n_{\mathrm{D}, \text { div }}\left(10^{19} \mathrm{~m}^{-3}\right)$ & 0.18 & 0.18 & 0.46 & 0.59 & 8.2 & 7.7 & - \\
\hline$n_{\mathrm{D}_{2}, \text { div }}\left(10^{19} \mathrm{~m}^{-3}\right)$ & 0.36 & 0.39 & 1.3 & 1.3 & 32. & 26. & - \\
\hline$T_{\mathrm{D}, \text { div }}(\mathrm{eV})$ & 3.2 & 3.2 & 2.6 & 2.4 & 2.0 & 2.0 & - \\
\hline$\Gamma_{\text {rocyc }}\left(10^{22} \mathrm{~s}^{-1}\right)$ & 5.40 & 5.40 & 5.36 & 5.36 & 5.29 & 5.29 & - \\
\hline$\Gamma_{\text {rcom }}\left(10^{22} \mathrm{~s}^{-1}\right)$ & 0.054 & 0.053 & 0.50 & 0.38 & 40. & 18. & - \\
\hline$\Gamma_{\mathrm{D}, \text { ion }}\left(10^{22} \mathrm{~s}^{-1}\right)$ & 5.0 & 4.7 & 5.4 & 5.1 & 44. & 24. & - \\
\hline$\Gamma_{\mathrm{D}_{2} \text {,ion }}\left(10^{22} \mathrm{~s}^{-1}\right)$ & 0.44 & 0.74 & 0.50 & 0.68 & 1.9 & -0.84 & - \\
\hline$\Gamma_{\text {MAR }}\left(10^{22} \mathrm{~s}^{-1}\right)$ & - & 0.066 & - & 0.10 & - & 2.7 & - \\
\hline
\end{tabular}

By particle conservation, $\Gamma_{\text {rccyc }}+\Gamma_{\text {rccom }}=\Gamma_{D, \text { ion }}+\Gamma_{D_{2} \text {,ion }}$, where $\Gamma_{\text {rccyc }}$ is the recycling rate (ion flux to targets), $\Gamma_{\text {recom }}$ is the recombination rate (both are input to DEGAS 2), $\Gamma_{\mathrm{D} \text {,ion }}$ is the ionization rate of atoms, and $\Gamma_{\mathrm{D}_{2} \text {,ion }}$ is the rate of ion generation from molecular dissociation. In the model II runs, the rate of atoms generated by MAR, $\Gamma_{\mathrm{MAR}}$, enters implicitly as a negative contribution to $\Gamma_{\mathrm{D}_{2} \text {,ion }}$ and is balanced by an equal positive contribution to $\Gamma_{\mathrm{D} \text {,ion }}$.

In plasma model $\mathrm{A}$, the electron temperature is greater than $5 \mathrm{eV}$ throughout the divertor plasma. Neither recombination nor MAR are important. The lower $D_{\alpha}$ in $A . I I$ is the result of a smaller number of Balmer- $\alpha$ photons per ionization in model II for $T>3 \mathrm{eV}$. In both A.I and A.II, molecules are predominantly broken up in regions with $T \simeq 5-10 \mathrm{eV}$. Over this range, model II yields more ions per molecule, leading to the larger $\Gamma_{\mathrm{D}_{2} \text {,ion }}$ in A.II.

With the detached 1-2 eV region, plasma model B yields significant re- 
combination and MAR. Recombination is in fact observed experimentally; it has been found that the number of ions lost to recombination can be comparable to the number striking the divertor plates[11]. However, in B.I and B.II, recycling is still dominant, and the results are qualitatively similar to A.I and A.II.

In plasma model $\mathrm{C}$, the factor of two reduction in temperature relative to model $\mathrm{B}$ leads to recombination rates which are more than three times the recycling source. In C.II, MAR contributes another $15 \%$ to the source of recombined atoms. The sink of ions due to MAR exceeds the rate at which they are created from $D_{2}$ dissociation; hence, $\Gamma_{D_{2} \text {,ion }}<0$. Again, the drop in the $\mathrm{D}_{\alpha}$ rate between C.I and C.II results from the lower conventional recombination rate and the higher number of the photons / ionization in model II. The other differences between the C.I and C.II results arise predominantly from the lower rate of recombination predicted by model II. The spatial distribution of the $\mathrm{D}_{\alpha}$ emission is shown in Fig. 2; Fig. 3 presents a tomographic reconstruction of the measured $\mathrm{D}_{\alpha}$ emission for comparison. Both show peaks in the detached region near the outer target where recombination is suspected to occur.

For C.I and C.II, the divertor pressures are comparable to the measured values. The global Balmer- $\alpha$ emission rates exceed the measured rate, however. Obtaining better agreement will require improvements to both the plasma and atomic physics models. The present approach to modeling the plasma would benefit from the addition of more experimental data regarding the private flux region plasma. But, given the strong dependence of recombination on plasma temperature and density, a self-consistent coupling of DEGAS 2 and CRAMD with a 2-D edge plasma code may be needed to match the data. The next steps in refining the DEGAS 2 atomic physics would be the inclusion of ion-neutral and neutral-neutral elastic scattering.

\section{ACKNOWLEDGMENTS}

This work was supported by U.S. Department of Energy Contracts No. DE-AC02-76-CHO-3073 and DE-FG02-91-ER-54109. The authors wish to acknowledge useful discussions with J. Kesner.

\section{REFERENCES}

[1] JANESCHITZ, G., et al., J. Nucl. Mater. 220-222 (1995) 73.

[2] STOTLER, D. P., KARNEY, C. F. F., Contrib. Plasma Phys. 34 (1994) 392.

[3] HELANDER, P., et al., Phys. Plasmas 1 (1994) 3174.

[4] PIGAROV, A. Yu., KRASHENINNIKOV, S. I., Application of CollisionalRadiative, Atomic-Molecular Model to the Recombining Divertor Plasma, Phys. Lett. A (in press).

[5] HUTCHINSON, I. H., et al., Phys. Plasmas 1 (1994) 1511.

[6] HEIFETZ, D.B., "Neutral Particle Transport", Physics of Plasma-Wall Interactions in Controlled Fusion, (POST, D.E., BEHRISCH, R., Eds.), 


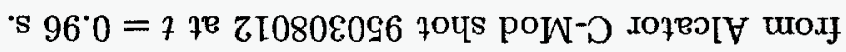

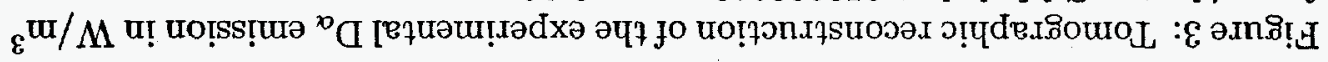

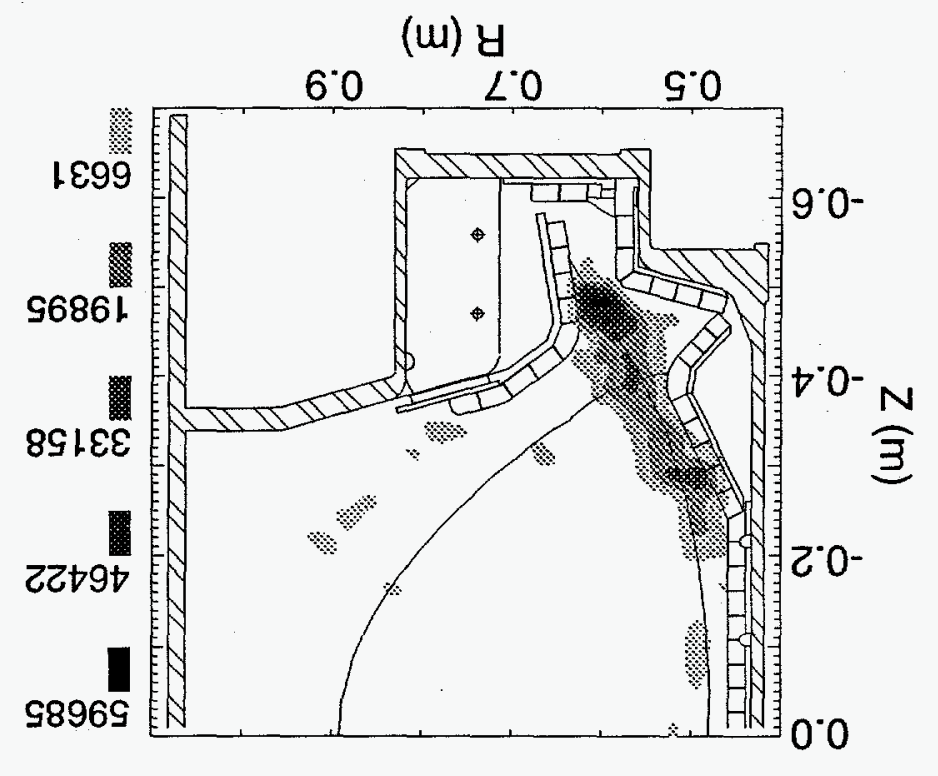

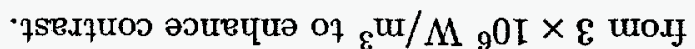

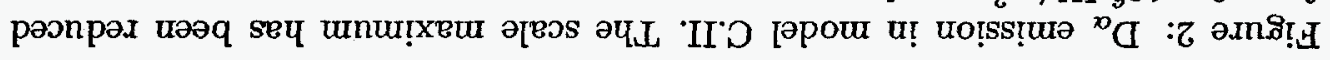

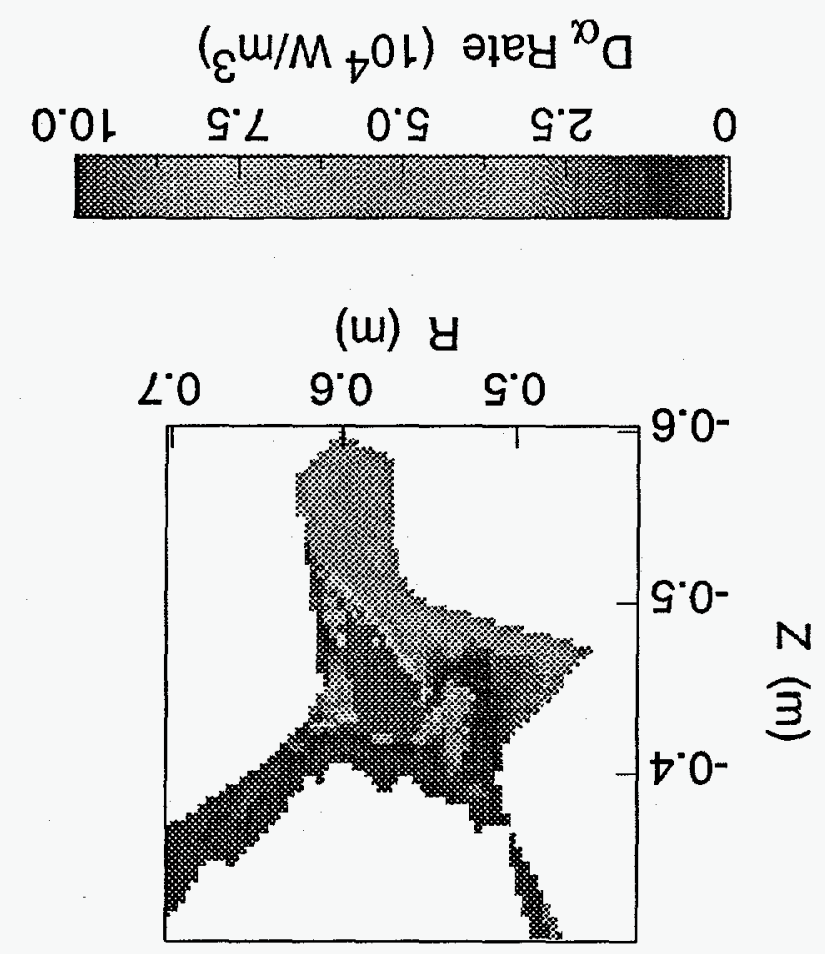


Plenum Press, New York (1986) 595-771.

[7] STOTLER, D. P., et al., in Plasma Physics and Controlled Nuclear Fusion Research 1994 (Proc. 15th Int. Conf. Seville, 1994), Vol. 3, IAEA, Vienna (1996) 363.

[8] KESNER, J., Phys. Plasmas 2 (1995) 1982.

[9] NIEMCZEWSKI, A., Neutral Particle Dynamics in the Alcator C-Mod Tokamak, PhD Thesis, Massachusetts Inst. of Technology, Cambridge (1995).

[10] STANGEBY, P. C., Plasma Phys. Control. Fusion 37 (1995) 1031.

[11] LUMMA, D., et al., Radiative and 3-Body Recombination in the Alcator C-Mod Divertor, submitted to Phys. Plasmas. 